\title{
The significance of magnetic resonance imaging in severe femoral trochlear dysplasia assessment
}

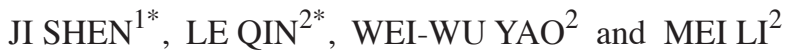 \\ Departments of ${ }^{1}$ Orthopaedics and ${ }^{2}$ Radiology, Shanghai Jiao Tong University Affiliated Sixth People's Hospital, \\ Shanghai 200233, P.R. China
}

Received July 7, 2016; Accepted April 10, 2017

DOI: 10.3892/etm.2017.5217

\begin{abstract}
The clinical diagnosis of femoral trochlear dysplasia primarily relies on imaging. In the past, plain imaging was the major source of diagnosis. The present study investigated the application of magnetic resonance imaging (MRI) in the objective assessment of severe femoral trochlear dysplasia. A retrospective analysis was performed on knee MRIs from 30 normal subjects (30 knees) and 59 patients (61 knees) with severe femoral trochlear dysplasia based on the Dejour morphological classification. Cartilage and subchondral bone landmarks were used to compare a series of measurements between patient and control groups. These measurements included the femoral trochlear groove depth, sulcus angle, the lateral trochlear inclination, trochlear facet asymmetry, the femoral medial and lateral condyle symmetry, and the ratios between the femoral medial/lateral condyles and the maximal trochlear width. The measurement values based on the two types of landmarks were also compared within the patient and control groups, separately. In addition, the femoral trochlear groove depth, sulcus angle, lateral trochlear inclination and trochlear facet asymmetry of patients with different Dejour types were compared. Significant differences were observed in the femoral trochlear groove depth, sulcus angle, lateral trochlear inclination and trochlear facet asymmetry between the patient and control groups $(\mathrm{P}<0.05)$. Based on the two types of landmark, all indexes were significantly different $(\mathrm{P}<0.05$; with the exception of lateral trochlear inclination) between the patient and control groups. Among patients with various Dejour types, the femoral trochlear groove depth, sulcus angle, lateral trochlear inclination and trochlear facet asymmetry demonstrated no significant differences. MRI exhibited
\end{abstract}

Correspondence to: $\mathrm{Dr} \mathrm{Mei} \mathrm{Li}$, Department of Radiology, Shanghai Jiao Tong University Affiliated Sixth People's Hospital, 600 Yishan Road, Shanghai 200233, P.R. China

E-mail: limei6th@sina.com

${ }^{*}$ Contributed equally

Key words: femoral trochlear dysplasia, magnetic resonance imaging, assessment advantages in revealing articular cartilage over conventional radiography and computed tomography. Therefore, cartilage landmarks in MRI images may be utilized to objectively evaluate femoral trochlear dysplasia in patients with severe femoral trochlear dysplasia.

\section{Introduction}

As one of the major pathological causes of patellar dislocation, femoral trochlear dysplasia has an incidence of $85-96 \%(1,2)$, and is typically caused by genetic factors (3). Under normal conditions, when a knee joint flexes $20^{\circ}$ from the fully extended position, the patella is only able to shift inward by $5 \mathrm{~mm}$ (4). Only when the flexion reaches $20-30^{\circ}$ do the patellofemoral joints align perfectly (4). Morphological changes of the femoral trochlea in these patients may cause abnormal patella movement and lead to patella dislocation (5). The clinical diagnosis of femoral trochlear dysplasia primarily relies on imaging diagnosis. Historically, plain imaging has been the major source of diagnosis (6). A sulcus angle of $>150^{\circ}$ on an axial radiograph is considered to indicate femoral trochlear dysplasia (6). However, in axial radiographs, the trochlear groove displayed is located on the distal side of the femoral trochlea, which does not represent the true morphology of the proximal trochlea (6). As such, the reliability of this method was considerably low (6). Furthermore, in $96 \%$ of patients with trochlear dysplasia, lateral radiography revealed the femoral trochlear groove crossing through the medial and lateral femoral condylar bumps, appearing as a 'crossing sign' or 'supratrochlear spur' when the medial and lateral femoral condyles overlapped (7). This imaging method has several limitations, including the required scanning posture and difficulties in attaining quantitative measurements (8-10).

A study by Dejour and Le Coultre (7) classified femoral trochlear morphologies into four types: (A) Normal trochlear shape, but shallow trochlear groove; (B) markedly flattened or even convex trochlea; (C) trochlear facet asymmetry, with an overly high lateral facet and a hypoplastic medial facet; and (D) type $\mathrm{C}$ features and vertical links between facets or cliff pattern. Computed tomography (CT) is not able to reveal articular cartilage, and so the femoral trochlea observed on CT images based on the bone landmarks does not reflect its true morphology. In contrast, magnetic resonance imaging (MRI) has the advantages of projecting articular cartilage 
and the surrounding ligaments and muscles, thus this imaging modality has become the basic method for evaluating femoral trochlear dysplasia. However, regardless of whether CT or MRI is used, due to the variety and complicated morphological structures of the proximal trochlea, multiple deepest points of the trochlear groove have often been observed when diagnosing severe trochlear dysplasia (types B-D), causing difficulties in confirming the desired anatomical landmarks (8). This ambiguity reduces measurement reliability and complicates the preoperative diagnosis. Previous studies have reported multiple methods by which the deepest point of the trochlear groove may be located from MRIs $(9,11)$. However, whether these methods are able to accurately find the anatomic landmark and correctly diagnose trochlear dysplasia remains uncertain (11). The aim of the present study was to determine the measurement values of severe trochlear dysplasia acquired from MRI and to investigate the application values of MRI, such that more evidence may be gained for clinical assessment, and to determine the differences in measurements between several trochlear dysplasia patients and normal subjects.

\section{Subjects and methods}

Patients. A total of 59 patients (61 knees) from the Shanghai Jiao Tong University Affiliated Sixth People's Hospital (Shanghai, China) who demonstrated symptoms of patellar instability between January 2013 and May 2014 were enrolled in the present study, and 30 healthy volunteers were enrolled as the control group. The patient inclusion criteria were a medical history of $>1$ patellar dislocation prior to admission and CT images of knees suggesting severe trochlear dysplasia as Dejour types B-D, which were determined according to the Dejour trochlear dysplasia system (7). Exclusion criteria were as follows: Osteoarthritis, meniscus tears, metabolic bone disease, rheumatoid arthritis and a history of ipsilateral knee surgery. There were 19 males and 40 females in the patient groups, with an age range of 14-44 years (mean, 23.6 years). There were 31 cases in the left knee and 30 cases in the right knee. The present study was approved by the Ethics Committee of Shanghai Jiao Tong University Affiliated Sixth People's Hospital and all patients provided informed consent.

CT examination. All patients lay on their backs with their knee fully extended whilst a plain CT scan was performed using a LightSpeed VCT CT scan system (64 slices; GE Healthcare, Chicago, IL, USA). The slice thickness and slice spacing were both $5 \mathrm{~mm}$. The tube current was set to $220 \mathrm{~mA}$, and the tube voltage was set to $120 \mathrm{kV}$. Slice reconstruction was based on 0.625-mm-thick slices.

MRI examination and measurement method. MRI examinations were performed with a Philips Intera-Achieva 3.0T system with a standard knee coil (Philips Medical Systems, Inc., Bothell, WA, USA). Patients and healthy volunteers lay on their backs with knee flexion of $20-30^{\circ}$. The major imaging techniques included cross sectional fast spin-echo T2 weighted image fat-suppressed sequences, with a slice thickness of $3 \mathrm{~mm}$ and a slice spacing of $0.3 \mathrm{~mm}$. The most proximal images revealing the complete medial and lateral facet cartilage of trochlea on craniocaudal MRI slices were selected (Figs. 1 and 2). Cartilage and subchondral bone landmarks were used to compare a series of measurements, including the femoral trochlear groove depth, the sulcus angle, the lateral trochlear inclination, trochlear facet asymmetry, the femoral medial and lateral condyle symmetry, and the ratios between the femoral medial/lateral condyles and the maximal trochlear width (Fig. 3). The following comparisons were made: i) The differences in measurements between patient and control groups based on cartilage and subchondral bone landmarks; ii) differences in measurements based on cartilage and subchondral bone landmarks within the patient and control groups; and iii) differences in the sulcus angle, trochlear groove depth, the lateral trochlear inclination and trochlear facet asymmetry among patients with types B, C and D trochlear dysplasia. Measurement values were independently determined by an orthopaedic physician and a senior musculoskeletal radiologist blinded to patient clinical data.

Statistical analysis. All statistical analysis was performed using SPSS 19.0 software (IBM Corp., Armonk, NY, USA). All data was represented as mean \pm standard error. Independent sample t-test was used to compare the difference between the patient group and the control group. Paired sample t-test was applied to the comparison between the measurements by cartilage and subchondral bone landmark. Kruskal-Wallis test was used to compare measurements among patients with different types of trochlear dysplasia. Intraclass correlation coefficient was used to compared the inter-observer reliability $(<0.2$, poor; 0.2-0.4, moderate; 0.4-0.6, fair; 0.6-0.8, good; 0.8-1.0, very good). $\mathrm{P}<0.05$ was considered to indicate a statistically significant difference.

\section{Results}

Significant differences were observed in the femoral trochlear groove depth, sulcus angle, lateral trochlear inclination and trochlear facet asymmetry between the patient and control groups $(\mathrm{P}<0.05$; Table I). However, no significant differences were observed between the two groups in terms of the femoral medial and lateral condyle symmetry and the ratios between the femoral medial/lateral condyles and the maximal trochlear width (Table I). When comparing within-group results, all indices based on the two landmarks in the patient group, with the exception of the lateral trochlear inclination $(\mathrm{P}=0.085)$, were significantly different $(\mathrm{P}<0.05$; Table II). All measurements in the control group were statistically different $(\mathrm{P}<0.05$; Table II). No significant differences were observed between patients with Dejour types B, C and D, in the regard of sulcus angle $\left(\mathrm{x}^{2}=3.348 ; \mathrm{P}=0.187\right)$, trochlear groove depth $\left(\mathrm{x}^{2}=1.809\right.$; $\mathrm{P}=0.405)$, lateral trochlear inclination $\left(\mathrm{x}^{2}=1.921 ; \mathrm{P}=0.383\right)$ and trochlear facet asymmetry $\left(\mathrm{x}^{2}=1.363 ; \mathrm{P}=0.506\right)$. In the observer credibility evaluation, the intraclass correlation coefficient of a single measurement and the mean measurement value was between good and very good (0.691-0.869; $\mathrm{P}<0.05)$.

\section{Discussion}

Patients with recurrent patellar dislocation often have pathological origins of patellar instability, including trochlear dysplasia, increased Tibial Tuberosity-Trochlear Groove 
A

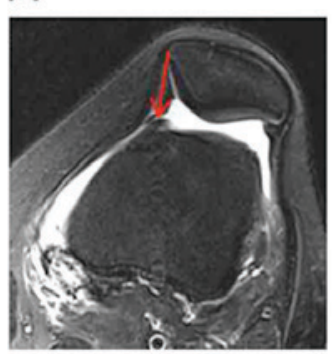

E

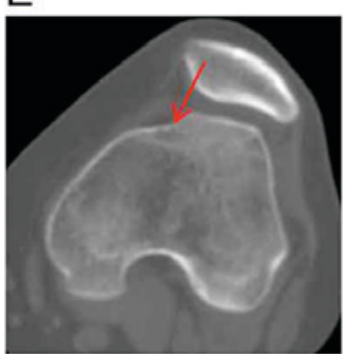

B

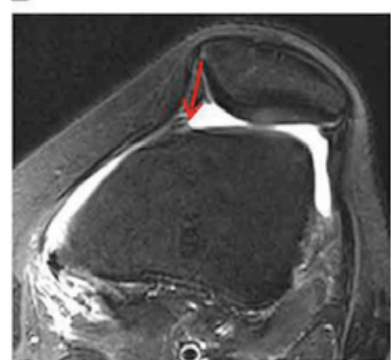

F

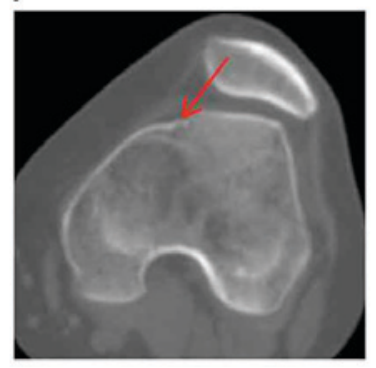

C

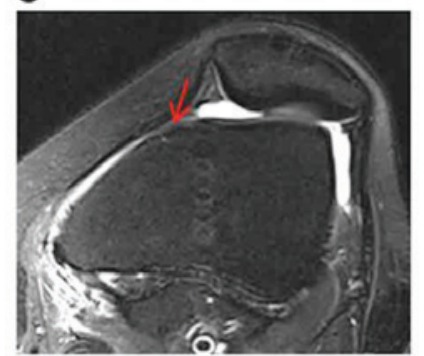

G

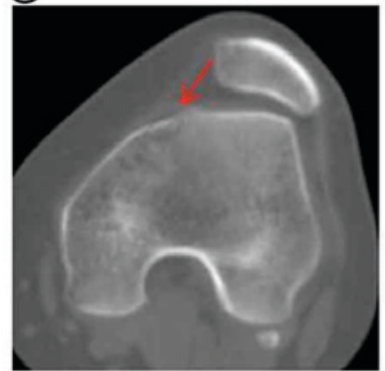

D

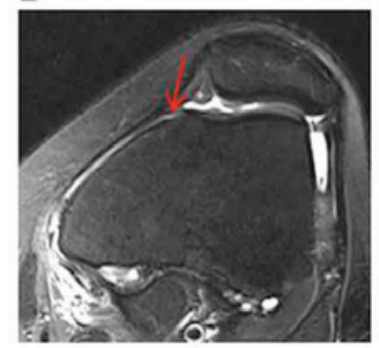

$\mathrm{H}$

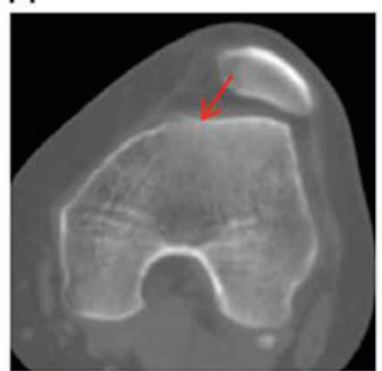

Figure 1. Images from a 26-year-old female with type D femoral trochlear dysplasia. (A-D) Continuous sectional magnetic resonance images from the same patient (cross-sectional fat-suppressed fast spin-echo T2 weighted image sequence). (E-H) Computed tomography plain scan cross-sectional images with corresponding femoral trochlear joint facet bone morphologies. Due to severe femoral trochlear dysplasia, a cliff pattern alteration and multiple deepest points are observed on the femoral trochlear lateral facet (red arrows).

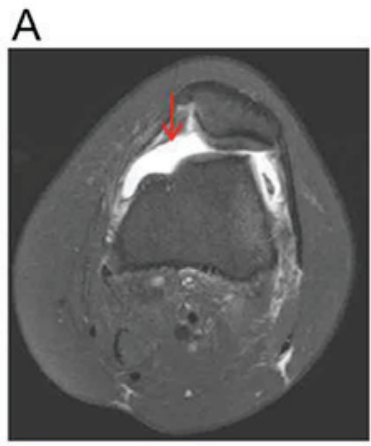

B

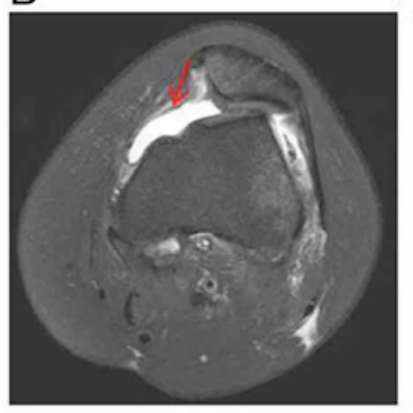

C

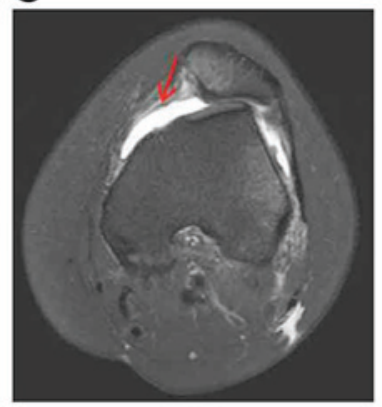

D

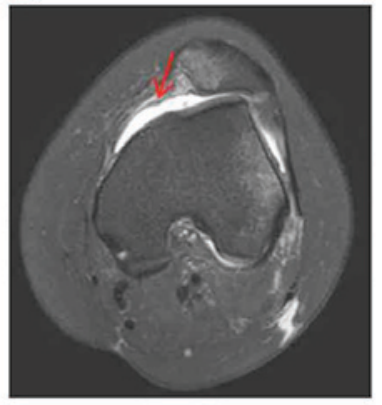

Figure 2. Images from a 20-year-old female with type D femoral trochlear dysplasia. (A-D) Continuous sectional magnetic resonance images from the same patient (cross-sectional fat-suppressed fast spin-echo T2 weighted image sequence). Based on the bone landmarks, multiple trochlear deepest points are present (red arrows). Based on the cartilage landmark, the trochlear deepest point revealed in image D was selected as the anatomic landmark. The cartilage on the medial facet is clearly apparent at the distal layer to the lateral facet.

distance, patella alta and patellar tilt (1). Among these pathologies, trochlear dysplasia has the highest incidence (1). In the present study, the purpose of acquiring measurements for the femoral trochlea was to clarify the fundamental factors of patellar dislocation and guide the surgical method and postoperative follow-up (4). An MRI cross-sectional sequence is important for the assessment of femoral trochlear dysplasia. A study by Stefanik et al (12) performed measurements on sections revealing the maximum posterior femoral condyle, whereas a study by Keser et al (13) acquired measurements on the section first revealing the lateral femoral trochlear cartilage on the craniocaudal image. However, it may be hypothesised that under normal circumstances the surface cartilage of the femoral trochlea would align with the cartilage of the patella, thus the observed cartilage surface may be closer to the true morphology of the femoral trochlea. We believed that whether or not the surface cartilage of the femoral trochlea is normal has a more profound effect on patellofemoral joint function. Therefore, the cross-section of the femoral trochlea at the craniocaudal positions of an MRI study that revealed the most proximal complete medial and lateral cartilage was chosen as the measurement section $(14,15)$. Unlike with CT imaging, the deepest point of the femoral trochlear groove and measurement layer is able to be determined based on the cartilage landmark with MRI in our patient group. In addition, on this layer, the subchondral bone landmark also displays the deepest point of the femoral trochlear groove.

The findings of the present study revealed that the results based on the two landmarks were the same between the patient and control groups. Except for the femoral medial and lateral condyle symmetry, and the ratios between the femoral medial and lateral condyles and the maximal trochlear width, all other measurements were statistically significant. The sulcus angles of the patient group measured in the present study were 
Table I. Intergroup comparisons of measurements between patient and control groups based on cartilage and subchondral bone landmarks.

\begin{tabular}{|c|c|c|c|c|}
\hline \multirow[b]{2}{*}{ Measurements } & \multicolumn{2}{|c|}{ Group } & \multirow[b]{2}{*}{$t$ value } & \multirow[b]{2}{*}{ P-value } \\
\hline & Patient & Control & & \\
\hline \multicolumn{5}{|l|}{ Measurements based on the cartilage landmark } \\
\hline Trochlear groove depth, mm & $2.4 \pm 1.1$ & $4.2 \pm 0.9$ & -7.959 & $<0.05$ \\
\hline Sulcus angle, degrees & $160.3 \pm 8.7$ & $146.9 \pm 4.1$ & 8.03 & $<0.05$ \\
\hline Trochlear facet asymmetry & $0.4 \pm 0.1$ & $0.5 \pm 0.1$ & -6.377 & $<0.05$ \\
\hline Lateral trochlear inclination, degrees & $13.7 \pm 5.6$ & $20.0 \pm 4.4$ & -5.371 & $<0.05$ \\
\hline Femoral medial and lateral condyle symmetry & $1.1 \pm 0.1$ & $1.1 \pm 0.0$ & -0.263 & 0.793 \\
\hline Ratio between the femoral medial condyle and the maximal trochlear width & $0.8 \pm 0.1$ & $0.8 \pm 0.1$ & 0.884 & 0.379 \\
\hline Ratio between the femoral lateral condyle and the maximal trochlear width & $0.8 \pm 0.1$ & $0.8 \pm 0.1$ & 1.757 & 0.082 \\
\hline \multicolumn{5}{|l|}{ Measurements based on the subchondral bone landmark } \\
\hline Trochlear groove depth, mm & $3.8 \pm 0.2$ & $6.0 \pm 0.2$ & -7.477 & $<0.05$ \\
\hline Sulcus angle, degrees & $149.2 \pm 1.0$ & $138.6 \pm 1.0$ & 7.589 & $<0.05$ \\
\hline Trochlear facet asymmetry & $0.4 \pm 0.1$ & $0.6 \pm 0.0$ & -6.434 & $<0.05$ \\
\hline Lateral trochlear inclination, degrees & $14.3 \pm 0.8$ & $22.5 \pm 0.7$ & -7.789 & $<0.05$ \\
\hline Femoral medial and lateral condyle symmetry & $1.0 \pm 0.0$ & $1.0 \pm 0.0$ & -1.373 & 0.173 \\
\hline Ratio between the femoral medial condyle and the maximal trochlear width & $0.7 \pm 0.0$ & $0.7 \pm 0.0$ & 1.367 & 0.175 \\
\hline Ratio between the femoral lateral condyle and the maximal trochlear width & $0.8 \pm 0.0$ & $0.8 \pm 0.0$ & 1.107 & 0.271 \\
\hline
\end{tabular}

Data are presented as the mean \pm standard error.

A
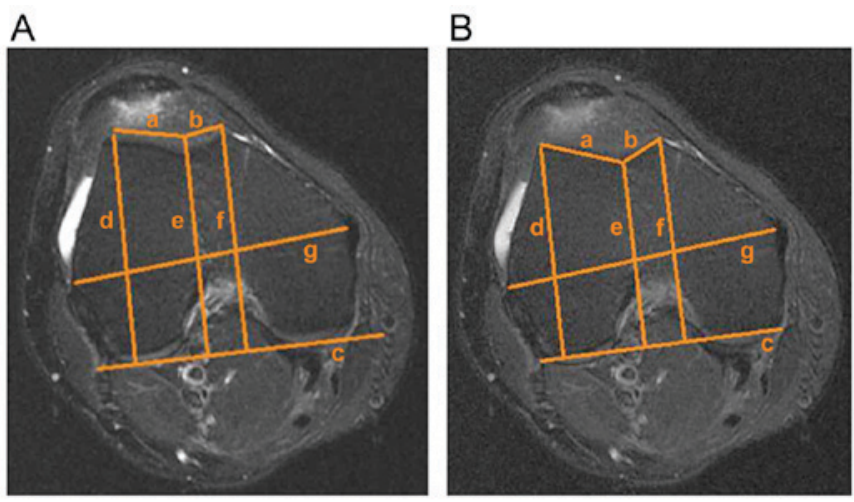

Figure 3. Fat-suppressed T2 weighted cross-sectional magnetic resonance imaging with a flexion of $20-30^{\circ}$. Measurements based on (A) cartilage and (B) subchondral bone landmarks. The sulcus angle is the included angle between a and $b$. The lateral trochlear inclination is the included angle between a and c. The depth of the femoral trochlear groove is -e; the asymmetry of the medial and lateral condyles of the femur is $\mathrm{d} / \mathrm{f}$; the trochlear facet asymmetry is b/a; the ratios between the heights of the femora medial/lateral condyles and the maximal width of the femoral trochlea are $\mathrm{f} / \mathrm{g}$ and $\mathrm{d} / \mathrm{g}$, respectively. a, the length of the lateral facet of the trochlea $\mathrm{b}$, the length of the medial facet of the trochlea; $\mathrm{c}$, the posterior condylar tangent; $d$, the height of the femoral lateral condyle; e, the distance between the deepest point of the femoral trochlea and the femoral posterior condylar tangent; f, the height of the femoral medial condyle; $g$, the maximal width of the femoral trochlea.

similar to the MRI results of $162.5^{\circ}$ reported in a study by Toms et al (16). However, the measurement value reported in a study by van Huyssteen et al (17) was $185^{\circ}$; this difference may be due to the fact that the authors only chose the layers displaying the lateral cartilage of the femoral trochlea with minor bumps. The layer used in the present study was able to reveal both medial and lateral cartilage, and the cartilage tissue appeared as small concavities. A femoral trochlear groove depth $<4 \mathrm{~mm}$ may be indicative of dysplasia (18). The measurements acquired in the present study were significantly smaller than the reference value and were similar to the $2.5 \pm 1.38 \mathrm{~mm}$ value reported in a study by Köhlitz et al (19). These results suggest that the femoral trochlear structure is flattened, resulting in poor alignment of the patellofemoral joint. Measurements of the sulcus angle and trochlear groove depth may serve as guides for the anteromedial tibial tubercle transfer technique for the treatment of patellar dislocation. A smaller sulcus angle and larger groove depth would be more likely to cause excess medialization of the tibial tubercle during surgery (19). This problem may cause a collision between the patella and the medial facet of the femoral trochlea, inducing pain (7). In the present study, assessment of the lateral trochlear inclination based on both landmarks yielded larger values than the results obtained in a study by Kamath et al (20), which reported values of $9.4^{\circ}$ and $8.8^{\circ}$ for the cartilage and subchondral landmarks, respectively. The reason for this discrepancy may be the different measurement layers used in these two studies; Kamath et al (20) used the layer displaying the maximal height of the lateral femoral condyle for measurements, whereas the measurements used in the present study were based on the cartilage landmark, and yielded similar results to those obtained in a study by Charles et al (18) $\left(13.31 \pm 1.36^{\circ}\right)$. This abnormal lateral trochlear inclination suggests that the antagonizing strength of the lateral facet of the femoral trochlea against the lateral translocation of the patella was weakened when the knee flexion 
Table II. Intragroup comparisons of measurements from patient and control groups based on cartilage and subchondral bone landmarks.

Landmark

Measurements

Cartilage Subchondral bone $t$ value P-value

\begin{tabular}{|c|c|c|c|c|}
\hline \multicolumn{5}{|l|}{ Measurements within the patient group } \\
\hline Trochlear groove depth, $\mathrm{mm}$ & $2.4 \pm 1.1$ & $3.8 \pm 0.2$ & -10.104 & $<0.05$ \\
\hline Sulcus angle, degrees & $160.3 \pm 8.7$ & $149.2 \pm 1.0$ & 12.075 & $<0.05$ \\
\hline Trochlear facet asymmetry & $0.4 \pm 0.1$ & $0.4 \pm 0.1$ & -3.522 & 0.001 \\
\hline Lateral trochlear inclination, degrees & $13.7 \pm 5.6$ & $14.3 \pm 0.8$ & -1.751 & 0.085 \\
\hline Femoral medial and lateral condyle symmetry & $1.1 \pm 0.1$ & $1.0 \pm 0.0$ & 6.363 & $<0.05$ \\
\hline Ratio between the femoral medial condyle and the maximal trochlear width & $0.8 \pm 0.1$ & $0.7 \pm 0.0$ & 11.491 & $<0.05$ \\
\hline Ratio between the femoral lateral condyle and the maximal trochlear width & $0.8 \pm 0.1$ & $0.8 \pm 0.0$ & 18.067 & $<0.05$ \\
\hline \multicolumn{5}{|l|}{ Measurements within the control group } \\
\hline Trochlear groove depth, $\mathrm{mm}$ & $4.2 \pm 0.9$ & $6.0 \pm 0.2$ & -8.649 & $<0.05$ \\
\hline Sulcus angle, degrees & $146.9 \pm 4.1$ & $138.6 \pm 1.0$ & 8.135 & $<0.05$ \\
\hline Trochlear facet asymmetry & $0.5 \pm 0.1$ & $0.6 \pm 0.0$ & -3.480 & 0.002 \\
\hline Lateral trochlear inclination, degrees & $20.0 \pm 4.4$ & $22.5 \pm 0.7$ & -3.372 & 0.002 \\
\hline Femoral medial and lateral condyle symmetry & $1.1 \pm 0.0$ & $1.0 \pm 0.0$ & 4.148 & $<0.05$ \\
\hline Ratio between the femoral medial condyle and the maximal trochlear width & $0.8 \pm 0.1$ & $0.7 \pm 0.0$ & 9.772 & $<0.05$ \\
\hline Ratio between the femoral lateral condyle and the maximal trochlear width & $0.8 \pm 0.1$ & $0.8 \pm 0.0$ & 14.299 & $<0.05$ \\
\hline
\end{tabular}

Data are presented as the mean \pm standard error.

reached $30^{\circ}$, resulting in patellar dislocation (21). A study by Teng et al (22) suggested that the lateral trochlear inclination was correlated with the incidence of patellar dislocation and emphasized the significance of the lateral facet of the trochlea in preventing patellar dislocation.

Studies regarding the association between the heights of the medial/lateral femoral condyles and femoral trochlear dysplasia have been rare. A study by Biedert and Bachmann (15) reported that patients with trochlear dysplasia had reduced absolute medial femoral condyle heights. However, the absolute values were significantly different among individuals. Therefore, in the present study ratios between the medial/lateral condyle heights were used, the same as the ratios between the femoral medial/lateral condyles and the maximal trochlear width, to exclude the interference of differences between individual cases. The results demonstrated that, in patients with severe femoral trochlear dysplasia, the lateral condyle was higher than the medial condyle. The results of the three indices were similar to those of the control group. These results suggested that femoral trochlear dysplasia should not alter the heights of the medial and lateral condyles, as significant changes were not observed in either height. The abnormal morphology of the joint facets was suggested as the major pathological appearance of trochlear dysplasia. A study by Van Haver et al (23) used the semi-automated landmark-based 3D image reconstruction technique and observed a higher medial condyle in the sagittal position compared with the control group, whereas the lateral condyle height was not significantly different. However, the authors selected the sagittal layers through the medial and lateral condyles of the femur, and so the changes in medial condyle height in different layers may vary.
In the present study, the proximal medial and lateral joint facets of the femoral trochlea were found to be covered in cartilage in the control group. However, in the patient group, only the lateral facet at the proximal femoral trochlea had cartilage coverage. In addition, the lateral facet exhibited a bump and cliff pattern alteration. The medial facet was observed as cortical bone. The cartilage of the medial facet was gradually visible distally. The simultaneous appearance of medial and lateral cartilage joint facets was only observed at the central part of the femoral trochlea at the craniocaudal position, and cartilage coverage at the medial facet was rare. At this point, the deepest point of the femoral trochlear groove could be identified. In addition, the missing medial cartilage at the proximal trochlea resulted in a higher position of the patellar joint cartilage compared with the trochlear joint facet, which is referred to here as 'relatively patellar alta'. The 'relatively patellar alta' resulted in a malalignment of the patellofemoral joint, which may induce patellar dislocation under minor external force. In the present study, the smaller trochlear facet asymmetry of patients compared with the control group also revealed abnormal morphology of the femoral trochlea. A study by Nelitz and Lippacher (14) identified a bump pattern in the proximal femoral trochlea in a comparative study using MRI and arthroscope techniques to assess patients with severe femoral trochlear dysplasia. Although the authors did observe a shallow trochlear groove, the initial position of the groove was near the far end, and the medial facet was accompanied by medial condylar dysplasia. Using CT scans, a study by Hing et al (24) also observed a negative correlation between the distance between the trochlear groove and the lateral facet, and the stability of the patella. However, MRI was 
able to demonstrate that a series of morphological changes were caused by femoral trochlear dysplasia. In the present study, according to such characteristic abnormal forms of the femoral trochlear, the layers displaying the deepest points of the femoral trochlea on MRI images were also identified on the corresponding CT images.

Intragroup comparisons revealed that, aside from the lateral trochlear inclination, all other indices based on cartilage and subchondral bone landmarks exhibited significant differences. Furthermore, all indices were statistically significant in the control group. This result confirmed that cartilage and subchondral bone morphologies were not perfectly matched in the patient or control groups. Application of the cartilage landmark was able to reveal the true morphology of the femoral trochlea. In the patient group, the existence of cartilage rendered the trochlear facet flatter and straighter, resulting in more severe dysplasia $(17,25)$. Unlike the results reported by Shih et al (25), in the present study the lateral trochlear inclination demonstrated no significant difference based on the cartilage and subchondral bone landmarks in the patient group, suggesting that the cartilage and subchondral bone surfaces on the lateral trochlear facet were almost parallel. Therefore, if the deepest point of the femoral trochlear groove determined by MRI was identified on the CT image, the measurement on the corresponding layer of the CT images may underestimate the severity of the femoral trochlear dysplasia. However, because the measurements of bony facets between patients and control groups were significantly different, this suggests that femoral trochlear dysplasia may still be determined using CT measurements in the initial diagnosis.

A further study of the B-D types of severe femoral trochlear dysplasia revealed no significant differences in the sulcus angle and trochlear depth, the lateral trochlear inclination or the medial and lateral joint facet asymmetry between types. This finding was similar to that reported in a study by Nelitz et al (9), which is possibly because the Dejour classification was determined by the proximal-side morphology of the femoral trochlea. Without complete cartilage coverage, the proximal trochlear is not able to form the patellofemoral joint. Therefore, in the present study, the selected layers were the central part of the trochlear at craniocaudal position. The different layers were the main reason that no correlation was observed between measurements acquired and Dejour's classification. A study by Fucentese et al (26) suggested that the preoperative classification of femoral trochlear dysplasia may predict the postoperative recovery of patients. They discovered that patients with types B and D trochlear dysplasia had better prognoses than patients with types $\mathrm{A}$ and $\mathrm{C}$ due to the reduced contact pressure between the patellofemoral joints in type B and D patients after surgery. The results of the present study suggested that the selected MRI image was able to objectively evaluate femoral trochlear dysplasia but not classify the type of dysplasia. It is worth noting that measurements of the selected layers may assist in evaluating trochlear dysplasia rather than replacing the classification of trochlear dysplasia. During preoperative assessment, the combination of femoral trochlear measurements and Dejour classification may provide more assistance in determining a surgical method and the prognosis.

In the present study, the parameters measured by MRI were able to reveal the pathological characteristics in patients with types B-D femoral trochlear dysplasia, including a flattened trochlear groove, flat joint facets and medial facet dysplasia. This method assisted with diagnosis and selecting the best surgical approach. In these patients, isolated force that strengthens the medial patellofemoral ligament for the medial translocation of the patella was not sufficient to counter the force of lateral dislocation caused by trochlear dysplasia. Therefore, combined femoral trochlear construction and medial patellofemoral ligament reconstruction were required to correct the dislocation $(27,28)$.

The present study was not without limitations. Further studies are required to confirm whether the determined deepest point of the femoral trochlear groove may be used for other measurements, such as the trochlear congruence angle and the Tibial Tuberosity-Trochlear Groove distance. In addition, although the credibility between observers ranged from good to very good in the present study, a study by Dornacher et al (29) suggested that, for type $C$ and $D$ patients with trochlear groove dysplasia, the correlation between the interclass and intraclass observers was not ideal. Therefore, larger sample sizes and experiments involving multiple institutions are required to confirm the reliability of these results. Finally, no comparative measurements were performed between corresponding layers from CT and MRI images, and it was not clear whether the results from both techniques were interchangeable.

MRI has unique advantages over X-ray and CT scans, such as no radiation exposure and the capacity to reveal cartilage and surrounding muscles and ligaments. The results of the present study suggested that, for patients with severe femoral trochlear dysplasia, MRI is able to determine the deepest point of the femoral trochlea for measurements. Measurements in the patient group were significantly different compared with the control group. Within the patient group, measurements based on the cartilage landmark were different from the measurements based on the subchondral bone landmark. Therefore, these objective measurements may assist in clinical assessment and treatment for such patients. However, the limitation of this method is that measurement values are unable to be used to determine the classification of the femoral trochlea, and so MRI measurements should be combined with morphological classifications.

\section{Acknowledgements}

The present study was supported by the National Natural Science Foundation of China (grant no. 81171312).

\section{References}

1. Earhart C, Patel DB, White EA, Gottsegen CJ, Forrester DM and Matcuk GR Jr: Transient lateral patellar dislocation: Review of imaging findings, patellofemoral anatomy, and treatment options. Emerg Radiol 20: 11-23, 2013.

2. Dejour H, Walch G, Nove-Josserand L and Guier C: Factors of patellar instability: An anatomic radiographic study. Knee Surg Sports Traumatol Arthrosc 2: 19-26, 1994.

3. Lippacher S, Reichel H and Nelitz M: Radiological criteria for trochlear dysplasia in children and adolescents. J Pediatr Orthop B 20: 341-344, 2011

4. Redziniak DE, Diduch DR, Mihalko WM, Fulkerson JP, Novicoff WM, Sheibani-Rad S and Saleh KJ: Patellar instability. Instr Course Lect 59: 195-206, 2010.

5. Botchu R, Obaid H and Rennie WJ: Correlation between trochlear dysplasia and the notch index. J Orthop Surg (Hong Kong) 21: 290-293, 2013 
6. Chhabra A, Subhawong TK and Carrino JA: A systematised MRI approach to evaluating the patellofemoral joint. Skeletal Radiol 40: 375-387, 2011

7. Dejour D and Le Coultre B: Osteotomies in patello-femoral instabilities. Sports Med Arthrosc 15: 39-46, 2007.

8. Tecklenburg K, Dejour D, Hoser C and Fink C: Bony and cartilaginous anatomy of the patellofemoral joint. Knee Surg Sports Traumatol Arthrosc 14: 235-240, 2006

9. Nelitz M, Lippacher S, Reichel H and Dornacher D: Evaluation of trochlear dysplasia using MRI: Correlation between the classification system of Dejour and objective parameters of trochlear dysplasia. Knee Surg Sports Traumatol Arthrosc 22: 120-127, 2014.

10. Smith TO, Cogan A, Patel S, Shakokani M, Toms AP and Donell ST: The intra- and inter-rater reliability of X-ray radiological measurements for patellar instability. Knee 20: 133-138, 2013.

11. Escala JS, Mellado JM, Olona M, Giné J, Sauri A and Neyret P: Objective patellar instability: MR-based quantitative assessment of potentially associated anatomical features. Knee Surg Sports Traumatol Arthrosc 14: 264-272, 2006

12. Stefanik JJ, Roemer FW, Zumwalt AC, Zhu Y, Gross KD, Lynch JA, Frey-Law LA, Lewis CE, Guermazi A, Powers CM and Felson DT: Association between measures of trochlear morphology and structural features of patellofemoral joint osteoarthritis on MRI: The MOST study. J Orthop Res 30: 1-8, 2012.

13. Keser S, Savranlar A, Bayar A, Ege A and Turhan E: Is there a relationship between anterior knee pain and femoral trochlear dysplasia? Assessment of lateral trochlear inclination by magnetic resonance imaging. Knee Surg Sports Traumatol Arthrosc 16: 911-915, 2008.

14. Nelitz M and Lippacher S: Arthroscopic evaluation of trochlear dysplasia as an aid in decision making for the treatment of patellofemoral instability. Knee Surg Sports Traumatol Arthrosc 22: 2788-2794, 2014

15. Biedert RM and Bachmann M: Anterior-posterior trochlear measurements of normal and dysplastic trochlea by axial magnetic resonance imaging. Knee Surg Sports Traumatol Arthrosc 17: 1225-1230, 2009.

16. Toms AP, Cahir J, Swift L and Donell ST: Imaging the femora sulcus with ultrasound, CT, and MRI: Reliability and generalizability in patients with patellar instability. Skeletal Radiol 38 329-338, 2009

17. van Huyssteen AL, Hendrix MR, Barnett AJ, Wakeley CJ and Eldridge JD: Cartilage-bone mismatch in the dysplastic trochlea. An MRI study. J Bone Joint Surg Br 88: 688-691, 2006.
18. Charles MD, Haloman S, Chen L, Ward SR, Fithian D and Afra R: Magnetic resonance imaging-based topographical differences between control and recurrent patellofemoral instability patients. Am J Sports Med 41: 374-384, 2013

19. Köhlitz T, Scheffler S, Jung T, Hoburg A, Vollnberg B, Wiener E and Diederichs G: Prevalence and patterns of anatomical risk factors in patients after patellar dislocation: A case control study using MRI. Eur Radiol 23: 1067-1074, 2013.

20. Kamath AF, Slattery TR, Levack AE, Wu CH, Kneeland JB and Lonner JH: Trochlear inclination angles in normal and dysplastic knees. J Arthroplasty 28: 214-219, 2013.

21. Balcarek P, Jung K, Ammon J, Walde TA,Frosch S, Schüttrumpf JP, Stürmer KM and Frosch KH: Anatomy of lateral patellar instability: Trochlear dysplasia and tibial tubercle-trochlear groove distance is more pronounced in women who dislocate the patella. Am J Sports Med 38: 2320-2327, 2010

22. Teng HL, Chen YJ and Powers CM: Predictors of patellar alignment during weight bearing: An examination of patellar height and trochlear geometry. Knee 21: 142-146, 2014.

23. Van Haver A, De Roo K, De Beule M, Van Cauter S, Audenaert E, Claessens T and Verdonk P: Semi-automated landmark-based 3D analysis reveals new morphometric characteristics in the trochlear dysplastic femur. Knee Surg Sports Traumatol Arthrosc 22: 2698-2708, 2014

24. Hing CB, Shepstone L, Marshall T and Donell ST: A laterally positioned concave trochlear groove prevents patellar dislocation. Clin Orthop Relat Res 447: 187-194, 2006.

25. Shih YF, Bull AM and Amis AA: The cartilaginous and osseous geometry of the femoral trochlear groove. Knee Surg Sports Traumatol Arthrosc 12: 300-306, 2004.

26. Fucentese SF, Zingg PO, Schmitt J, Pfirrmann CW, Meyer DC and Koch PP: Classification of trochlear dysplasia as predictor of clinical outcome after trochleoplasty. Knee Surg Sports Traumatol Arthrosc 19: 1655-1661, 2011.

27. Howells NR, Barnett AJ, Ahearn N, Ansari A and Eldridge JD: Medial patellofemoral ligament reconstruction: A prospective outcome assessment of a large single centre series. J Bone Joint Surg Br 94: 1202-1208, 2012.

28. Dejour D, Byn P and Ntagiopoulos PG: The Lyon's sulcus-deepening trochleoplasty in previous unsuccessful patellofemoral surgery. Int Orthop 37: 433-439, 2013.

29. Dornacher D, Reichel H and Lippacher S: Measurement of tibial tuberosity-trochlear groove distance: Evaluation of inter- and intraobserver correlation dependent on the severity of trochlear dysplasia. Knee Surg Sports Traumatol Arthrosc 22: 2382-2387, 2014. 